\title{
NOvA Muon Neutrino and Antineutrino Disappearance Results 2018
}

\author{
Dmitrii Torbunov \\ University of Minnesota
}

June 19, 2018

This document was prepared by [NOvA Collaboration] using the resources of the Fermi National Accelerator Laboratory (Fermilab), a U.S. Department of Energy, Office of Science, HEP User Facility. Fermilab is managed by Fermi Research Alliance, LLC (FRA), acting under Contract No. DE-AC02-07CH11359. 
- NOvA uses NuMI muon (anti)neutrino beam. Thank you Fermilab for this tremendous beam.

- The muon beam travels from the Near Detector at Fermilab to the Far Detector in northern Minnesota.

- As muons travel, some fraction of $\nu_{\mu}$ oscillates into $\nu_{e}$ and $\nu_{\tau}$.

- The muon neutrino disappearance analysis aims to determine $\left(\theta_{23}, \Delta m_{32}^{2}\right)$ oscillation parameters, by measuring the number of survived muon neutrinos at the Far Detector. 
Numu Analysis, Idea

- Muon neutrino oscillations produce a dip between 1-2 $\mathrm{GeV}$ range.

- Dip depth depends on the mixing angle $\theta_{23}$.

- Precise position of the dip depends on the $\Delta m_{32}^{2}$.

- But, presence of systematic uncertainties requires extra work to minimize their influence.

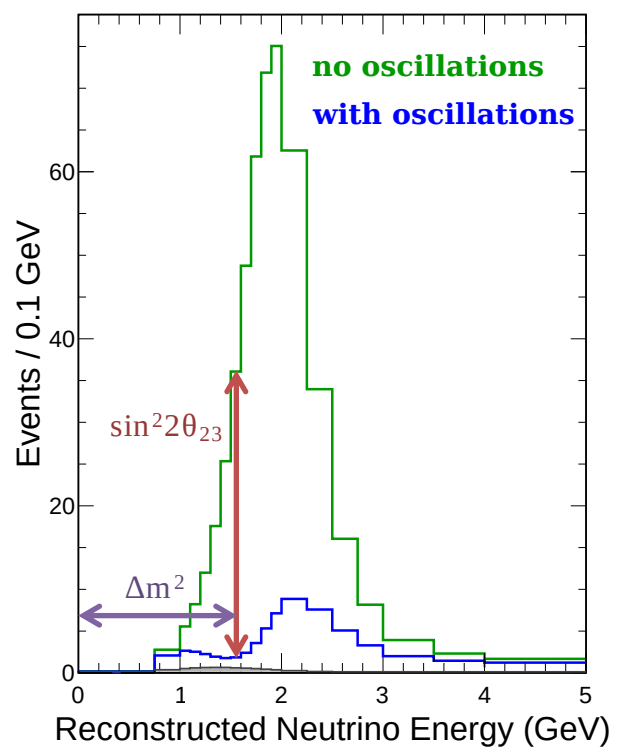


Numu Analysis 1, How to detect a muon neutrino?

- Muon neutrinos do not have electric charge, so they do not leave any tracks.

- We can only detect neutrinos if they happen to interact with the detector.

- The only such reaction, that allows us to identify a muon neutrino is a charged current interaction:
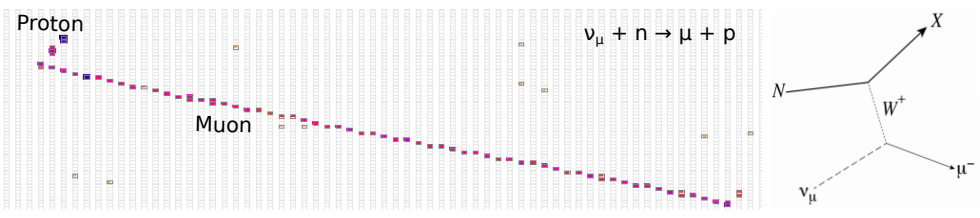


\section{Numu Analysis 2, Muon Neutrino selection 1}

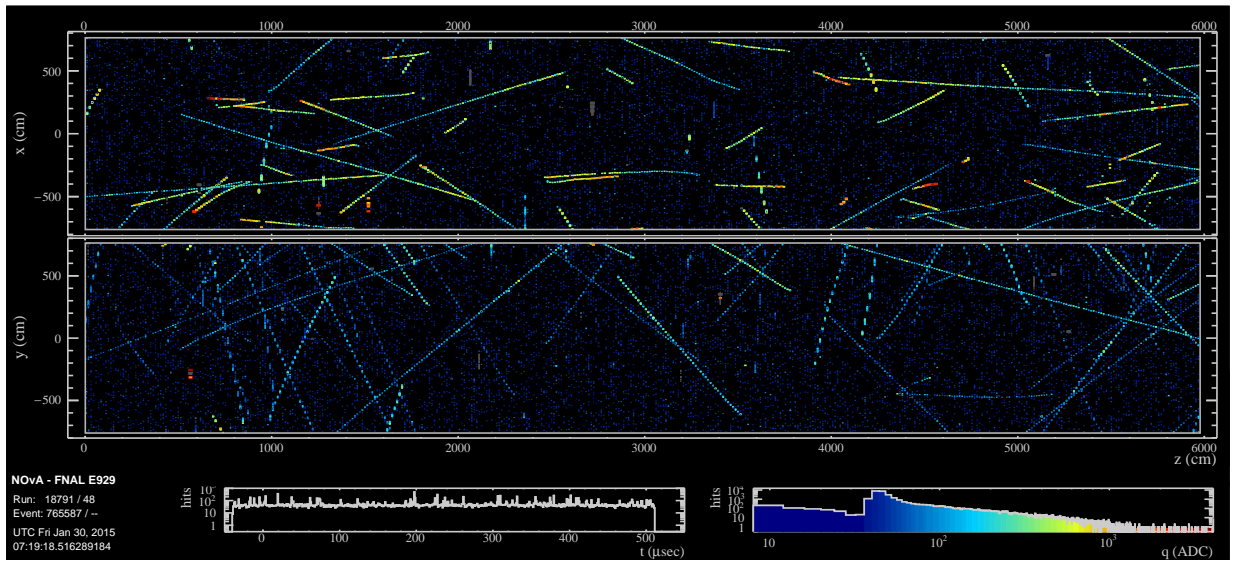

Where is a muon neutrino here? 
Numu Analysis 2, Muon Neutrino selection 2

- We identify the $\nu_{\mu} \mathrm{CC}$ event by the presence of the muon.

- First, we have a great computer vision algorithm CVN, which can identify particle types.

- But, we must also use another algorithm which looks at the physical properties of the tracks to distinguish muon tracks from pion tracks (which look similar).

- Finally, there are a lot of muons coming from the top cosmics. To minimize cosmics background we:

- use small timing window around the beam pulse

- veto muons that come from the edge of the detector

- have an algorithm that rejects the remaining muons, by analyzing their kinematics 


\section{Numu Analysis 2, Muon Neutrino selection 3}

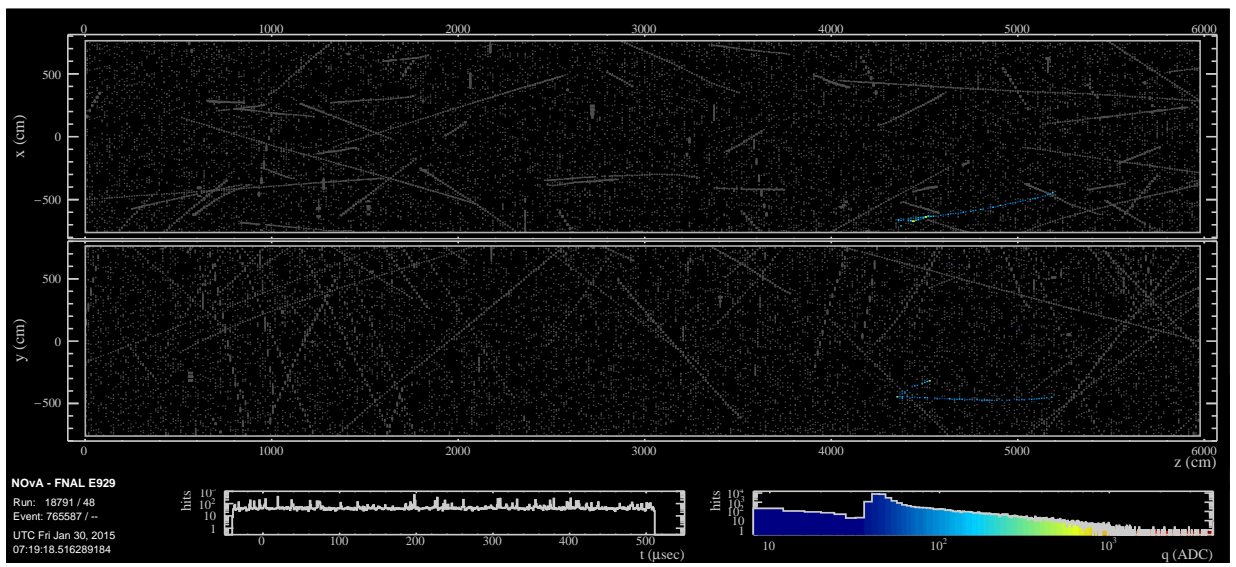

Here is a muon neutrino! 
Numu Analysis 3, Energy reconstruction

- Since we are looking at the $\nu_{\mu}$ CC events of the form:
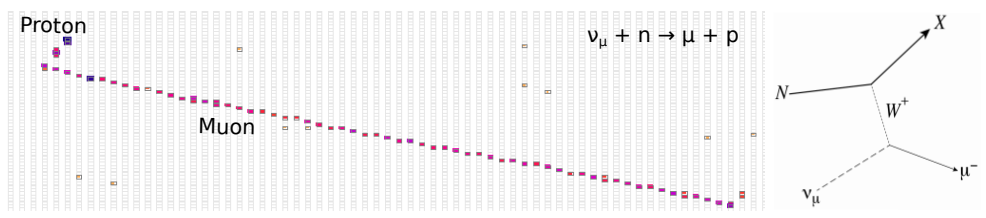

- The energy of the muon neutrino is

$$
E_{\nu_{\mu}}=E_{\mu}+E_{\mathrm{had}}
$$

- Energy of the muon $E_{\mu}$ is reconstructed from the muon's track length.

- Hadronic energy $E_{\text {had }}$ is reconstructed from the visible calorimetric energy. 
Numu Analysis 4, Quartiles split

- After, we have reconstructed neutrino's energies, we split our sample into 4 classes(also known at NOvA as "quartiles")

- The split is based on the fraction energy in the event, that comes from hadronic activity(i.e. not from muon)

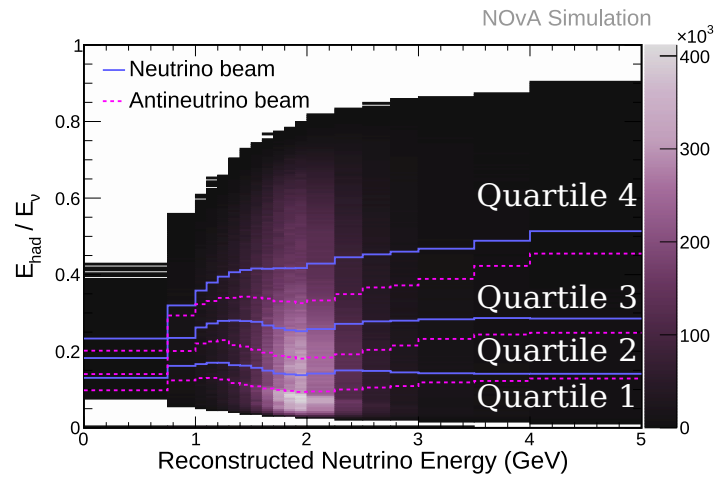

Quartile boundaries for muon (anti)neutrinos 
Numu Analysis 5, Exptrapolation

Finally, we use the Near Detector data to construct the Far Detector predicted energy spectrum:

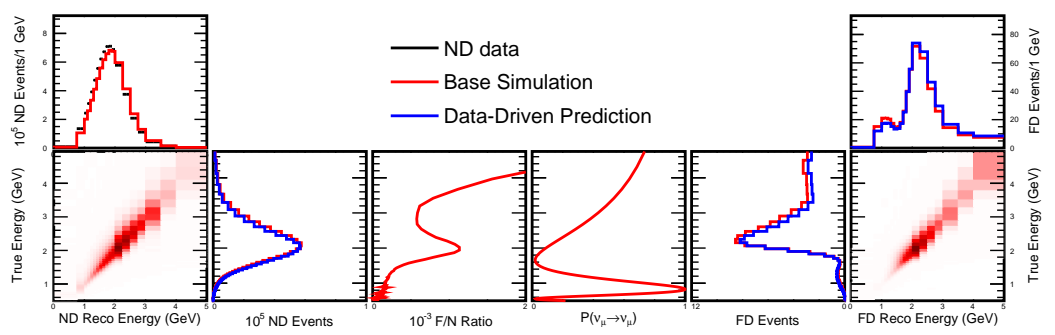

This extrapolation procedure helps us to minimize flux and cross-section uncertainties. 


\section{Numu Analysis 6, Data/Prediction comparison, Details}
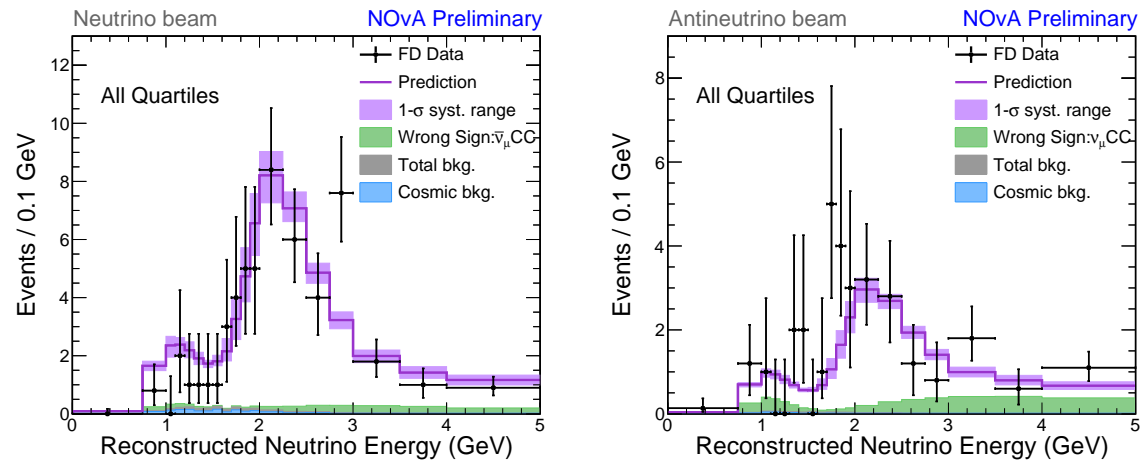

\begin{tabular}{|l|c|c|}
\hline Beam & Neutrinos & Antineutrinos \\
\hline Exposure & $8.9 \cdot 10^{20}$ POT & $6.9 \cdot 10^{20}$ POT \\
\hline Total Observed & 113 & 65 \\
\hline Best fit prediction & 121 & 50 \\
\hline Cosmic Bkgd. & 2.1 & 0.5 \\
\hline Beam Bkgd. & 1.2 & 0.6 \\
\hline Unoscillated & 730 & 266 \\
\hline
\end{tabular}

Prediction here is based on the best fit values for joint numu-disappearance and nue-appearance analysis 
Comparison with other experiments

NOvA Preliminary

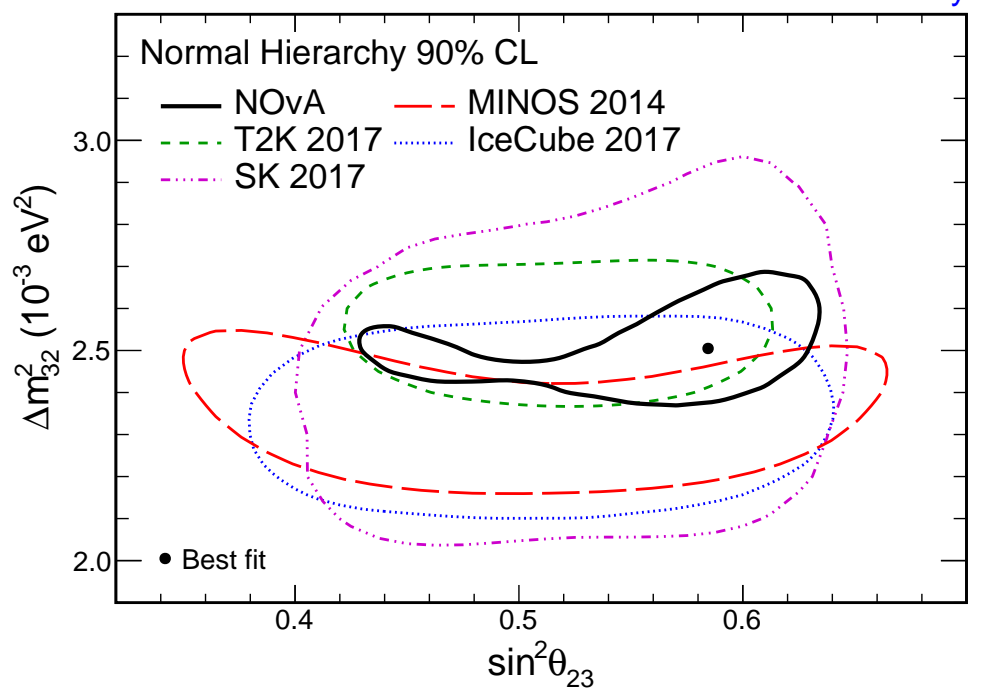

Best fit: $\sin ^{2} \theta_{23}=0.58 \pm 0.03, \Delta m_{32}^{2}=2.51_{-0.08}^{+0.12} \cdot 10^{-3} \mathrm{eV}^{2}$

Combined muon neutrino disappearance and electron neutrino appearance 
- We have the first results with neutrino and antineutrino data $\left(\sin ^{2} \theta_{23}=0.58 \pm 0.03, \Delta m_{32}^{2}=2.51_{-0.08}^{+0.12} \cdot 10^{-3} \mathrm{eV}^{2}\right)$.

- In the future we expect to refine our measurements of $\theta_{23}$ and $\Delta m_{32}^{2}$ by accumulating higher exposure values.

- This will improve NOvA's sensitivity to the mass hierarchy, $\theta_{23}$ octant and CP violation.

- Please find the full NOvA $\nu_{\mu}$ disappearance and $\nu_{e}$ appearance results in the next talk. 
Backups 
Data/Prediction comparison, Neutrinos

- We have observed 113 muon neutrino like events:

\begin{tabular}{|c|c|}
\hline Total Observed & 113 \\
\hline Best fit prediction & 121 \\
\hline Cosmic Bkgd. & 2.1 \\
\hline Beam Bkgd. & 1.2 \\
\hline Unoscillated & 730 \\
\hline
\end{tabular}

- Corresponding exposure $8.9 \cdot 10^{20}$ POT.

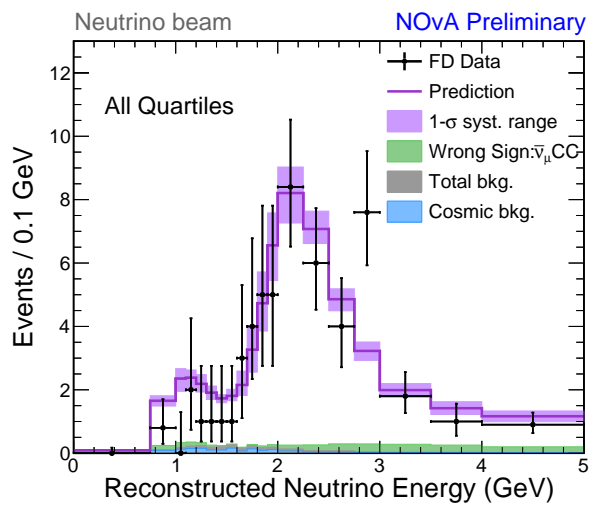


Data/Prediction comparison, Antineutrinos

- We have observed 65 muon antineutrino like events:

\begin{tabular}{|c|c|}
\hline Total Observed & 65 \\
\hline Best fit prediction & 50 \\
\hline Cosmic Bkgd. & 0.5 \\
\hline Beam Bkgd. & 0.6 \\
\hline Unoscillated & 266 \\
\hline
\end{tabular}

- Corresponding exposure $6.9 \cdot 10^{20}$ POT.

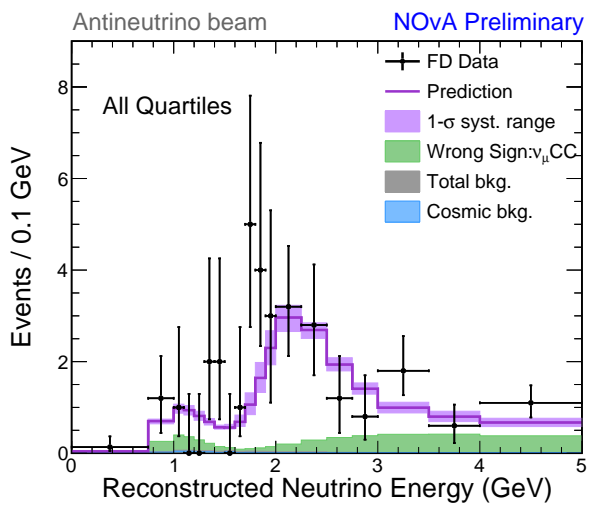


Numu Contours

Below are the contours that show $90 \%$ confidence contours for the oscillation parameters $\theta_{23}, \Delta m_{32}^{2}$

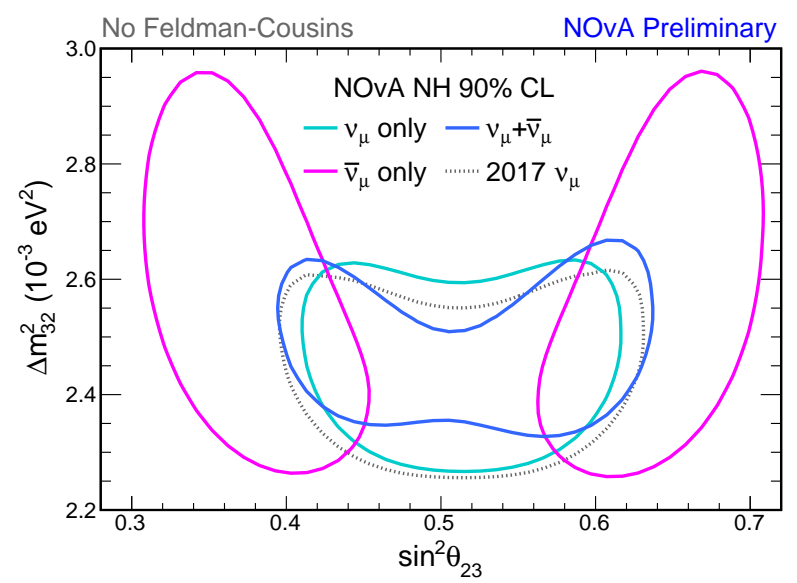

Consistency with the combined fit oscillation parameters for the neutrino and antineutrino datasets is better than $4 \%$. 
Octant-Hierarchy Sensitivity

Joint $\nu_{\mu}+\bar{\nu}_{\mu}$ fit prefers higher octant of $\theta_{23}$ for the normal hierarchy and lower octant for the inverted hierarchy.

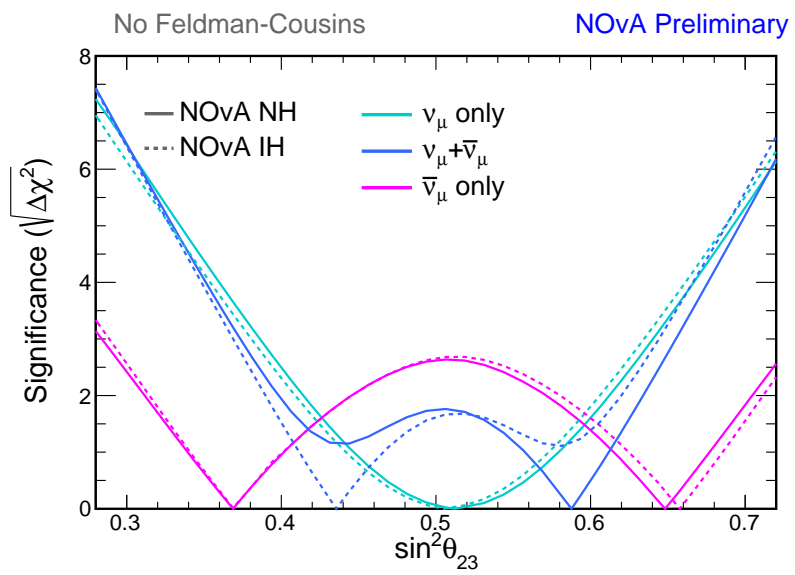


Numu Analysis 4, Motivation for Quartile split

Why do we split our sample in 4 parts?

- The NOvA's sensitivity towards oscillation parameters depends on how well we reconstruct neutrino energy.

- It turns out, that we can reconstruct muon's energy much better than the hadronic energy.

- So, by separating sample into 4 quartiles we can better exploit good energy reconstruction of the quartiles with low fraction of hadronic energy. 
Numu Analysis 4, Motivation for Quartile split 2

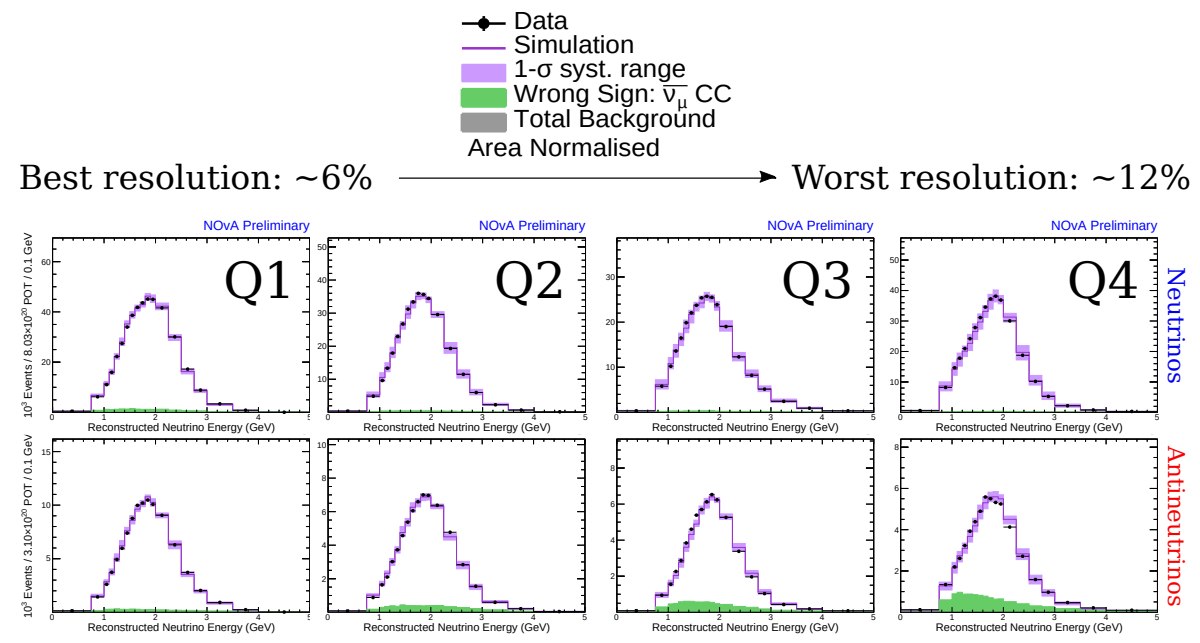

Near Detector Data and Simulation comparison.

All points are within $1-10 \%$. 
Numu Analysis 6, Data/Prediction comparison, Details
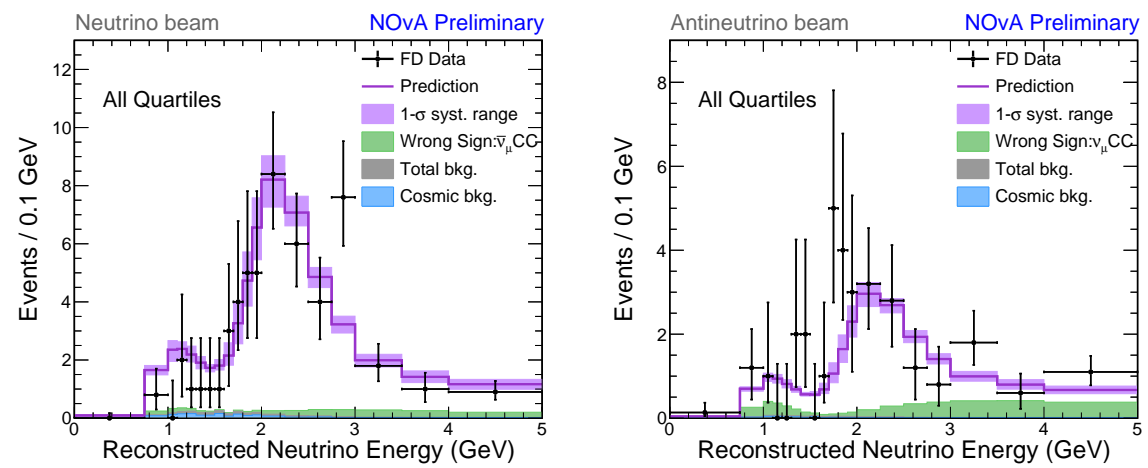

\begin{tabular}{|l|c|c|}
\hline Beam & Neutrinos & Antineutrinos \\
\hline Exposure & $8.9 \cdot 10^{20}$ POT & $6.9 \cdot 10^{20}$ POT \\
\hline Total Observed & 113 & 65 \\
\hline Best fit prediction & 121 & 50 \\
\hline Cosmic Bkgd. & 2.1 & 0.5 \\
\hline Beam Bkgd. & 1.2 & 0.6 \\
\hline Unoscillated & 730 & 266 \\
\hline
\end{tabular}

